Voix et Images

voixetimages

\title{
Les pseudonymes : " paravent derrière lequel se cachent des êtres méprisables " ou " mensonge qui ne fait de mal à personne "?
}

\section{Marie-Pier Luneau et Pierre Hébert}

Volume 30, numéro 1 (88), automne 2004

Le pseudonyme au Québec

URI : https://id.erudit.org/iderudit/009885ar

DOI : https://doi.org/10.7202/009885ar

Aller au sommaire du numéro

Éditeur(s)

Université du Québec à Montréal

ISSN

0318-9201 (imprimé)

1705-933X (numérique)

Découvrir la revue

Citer cet article

Luneau, M.-P. \& Hébert, P. (2004). Les pseudonymes : " paravent derrière lequel se cachent des êtres méprisables " ou « mensonge qui ne fait de mal à personne » ? Voix et Images, 30(1), 9-11. https://doi.org/10.7202/009885ar d'utilisation que vous pouvez consulter en ligne. 


\section{LES PSEUDON Y M S :}

" paravent derrière lequel se cachent des êtres méprisables" ou "mensonge qui ne fait de mal

à personne»?

\section{MARIE-PIER LUNEAU ET PIERRE HÉBERT}

Université de Sherbrooke

Le premier dictionnaire de pseudonymes québécois a été publié en 1936 par Francis Audet et Gérard Malchelosse. Dans l'esprit de leur époque, Audet et Malchelosse contribuaient ainsi «à l'édifice définitif de notre histoire nationale» en rétablissant l'ordre dans l'histoire littéraire, qui deviendrait vite «indéchiffrable si quelqu'un n'y veillait ${ }^{1} »$. Devant cette pratique subversive qu'est celle de la fausse signature, le rôle des critiques est bien de rétablir la vérité, au grand bénéfice de la postérité. Dans sa préface au dictionnaire, Aegidius Fauteux hésite toutefois. Il reconnaît que le pseudonyme peut parfois avoir servi de paravent à «des êtres méprisables»; cependant, il constate que ce délit mineur n'est plus qu'un mensonge «conventionnel» qui ne trompe guère «et surtout» ne fait «de mal à personne ${ }^{2}$ ».

Fauteux n'a pas tort. Les années vingt et trente foisonnent de noms de plume, ce qui l'amène à répéter que «jamais peut-être le pseudonyme ne s'est épanoui dans le jardin des lettres plus largement et plus librement qu'aujourd'hui ${ }^{3}$ ».

En apparence anodin, le discours des auteurs et du préfacier du premier dictionnaire de pseudonymes québécois pose bien les enjeux de cet objet d'étude. D'emblée, la critique oscille entre la condamnation sans appel et l'absolution, en fonction du degré de mensonge. C'est de bonne guerre: plus les critiques seront dupés, moins ils pardonneront à l'auteur fauteur de troubles. Mais l'histoire du Québec a justement ceci de particulier qu'elle compte très peu de mystifications littéraires à grand déploiement. On y cherchera vainement les Clotilde de Surville, les Ossian, les Émile Ajar. En comparaison avec ces grandes inventions d'écrivains mythiques, qui ont férocement entaché la crédibilité des «spécialistes» de la littérature, les tentatives des auteurs québécois pour déjouer la critique apparaissent bien timides. La plupart de ceux qui utilisent une fausse signature se nimbent d'un fugitif mystère, puis s'empressent de préciser leur identité, en conservant paradoxalement le nom de plume. On est conséquemment en droit de se demander à

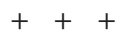

1 Ægidius Fauteux, «Préface», Francis J. Audet et Gérard Malchelosse, Pseudonymes canadiens, Montréal, G. Ducharme, libraire-éditeur, 1936, p. $10 . \quad 2$ Ibid., p. $8 . \quad 3$ Ibid., p. 7. 
quoi peuvent servir tous ces masques qui, dans les faits, ne masquent rien. Ces «mensonges honnêtes» sont-ils signes d'une difficulté, pour les auteurs, à assumer leur statut d'écrivain? Sont-ils, au contraire, gages de la bonne santé du champ littéraire, témoignant avec bonhomie d'une irrépressible propension au ludisme?

Mystification ou pas, l'évolution du régime de signatures des auteurs dans un corpus donné ne manque pas d'intérêt. On a en effet tendance à analyser exclusivement la question de la fausse signature en fonction de forces externes à la création littéraire - notamment la censure ou la critique - alors que cette pratique fait partie intégrante du processus d'écriture. Les multiples personnages découverts dans les malles de Fernando Pessoa sont autant de preuves de cette imbrication indissociable. Sous cet angle, la nouvelle signature incarne la liberté suprême que se donne le créateur en se nommant lui-même, ce qui n'implique pas nécessairement que cette liberté soit universellement bénéfique. La nouvelle naissance peut être, au mieux, libératrice ou sans effets. Au pire, elle est destructrice, lorsque son emprise sur l'identité première de l'écrivain devient trop grande ; on peut ainsi avancer qu'Émile Ajar a tué Romain Gary, contrairement au titre du témoignage posthume de Gary, Vie et mort d'Émile Ajar ${ }^{4}$. C'est d'abord et avant tout pour venir au monde, pour naître à la littérature, que les auteurs se sont servis de la fausse signature au Québec. Le pseudonyme est l'expédient par excellence qui représente l'auteur dans le monde des lettres, alors que l'individu reste sagement derrière, en retrait. Si «je» est un autre, tout devient possible, y compris le risque de dépossession de soi. Chose certaine, la connaissance des tenants et aboutissants du recours à la fausse signature exige une recherche et une réflexion dont ce numéro permettra à tout le moins de mesurer l'ampleur. En effet, on pourra y lire des articles qui proposent des éclairages diversifiés, afin de saisir le plus grand nombre de points de vue possible sur une pratique dont l'histoire reste à écrire.

Pour cette raison, le numéro s'ouvre sur un article de Marie-Pier Luneau qui propose un regard d'ensemble sur la pratique du pseudonyme au Québec. Elle nous offre ainsi un cadre à la fois heuristique et herméneutique qui éclaire la diversité des usages de la signature et de leurs significations. Cette étude couvre la période 1809-1979, mais les analyses qui suivent scrutent des pratiques singulières à partir du milieu du dix-neuvième siècle jusqu'au milieu du siècle suivant.

Les deux articles qui suivent abordent des cas exemplaires au dix-neuvième siècle, ceux de Pierre-Joseph-Olivier Chauveau et d'Henri-Raymond Casgrain. Lucie Robert propose une analyse de Chauveau, soulevant le problème de la multiplicité des identités sociales, en l'occurrence celui de la double carrière et de la signature. Elle y montre que le nom de plume et le pseudonyme, certes, distinguent l'écrivain de la personne civile, mais que cet usage est par ailleurs historicisé, c'est-à-dire utilisé «en fonction du rôle que l'écrivain accorde à la littérature et de la place que la littérature occupe dans la sphère publique». Pour ce qui est de Casgrain, Manon Brunet analyse un répertoire de vingt pseudonymes différents, en plus des quatre-

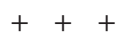

4 Romain Gary, Vie et mort d'Émile Ajar, Paris, Éditions Gallimard, 1981, 42 p. 
vingt-quatorze articles anonymes. L'intérêt, ici, est la mise en parallèle de la variation des signatures avec la trajectoire de l'auteur, littéraire certes, mais aussi idéologique: le Casgrain gaumiste laisse place à une figure moins connue, le libéraliste.

Chantal Savoie se penche sur l'écriture des femmes. Elle constate trois états en ce qui a trait à l'usage de la signature entre 1893 et 1929: le recours au prénom fictif, au nom complet fictif et au nom complet et véritable. On reconnaît Madeleine, Michelle Le Normand et Marie-Claire Daveluy, à titre d'exemples. Chantal Savoie entend "poser les bases d'une réflexion sur les signatures féminines en mettant en rapport les différentes formes de signatures féminines avec l'évolution de la trajectoire des femmes dans le champ littéraire, en tenant compte plus particulièrement des supports éditoriaux qui servent à diffuser leurs écrits, des genres littéraires qu'elles pratiquent, de même que de leur fortune littéraire à court et à long terme».

Quel est le lien entre le nom fictif et la transgression? Le cas de Louis Dantin paraît idéal pour comprendre cette question. Pierre Hébert propose une réflexion sur les stades qui mènent à la transgression en lien avec le recours soit à l'anonymat, soit à un nom fictif. On y suivra donc les différentes utilisations de la signature qui, en définitive, sont autant de procédés qui permettent à l'auteur, Louis Dantin, d'aller là où l'homme, Eugène Seers, refuse de se rendre. Distinction surannée? Pourtant, il faut peut-être distinguer l'homme de l'auteur construit afin de saisir toute la portée du nom fictif dans un contexte de transgression.

Patricia Godbout soulève un pan méconnu de la signature fictive, celle employée par les traducteurs. Pierre Daviault, Gérard Dagenais et John Glassco sont abordés successivement; enfin, le canular récent de David Solway, qui feint avoir traduit les poèmes d'Andeas Karavis, montre à quel point la signature, le traductionyme plus précisément, infléchit l'acte de lecture à partir de cas aussi divers qu'éclairants.

Tzvetan Todorov notait que la science qui avait pour but de décrire les continents a disparu une fois la tâche achevée. Autrement dit, les sciences particulières naissent dans l'obscurité et meurent dans la lumière. En ce qui concerne notre connaissance de la fausse signature, l'obscurité est encore très grande; toutefois, nous souhaitons que ce numéro définisse un continent encore mystérieux et que chacune des contributions fraie le chemin vers une meilleure compréhension de ce phénomène. 